\title{
The Integrated Sachs-Wolfe Effect in the Bulk Viscous Dark Energy Model
}

\author{
B. Mostaghel ${ }^{1}$, H. Moshafi ${ }^{2} \&$ S. M. S. Movahed ${ }^{1,2 \star}$ \\ 1 Department of Physics, Shahid Beheshti University, Velenjak, Tehran 19839, Iran \\ 2 Ibn-Sina Laboratory, Shahid Beheshti University, Velenjak, Tehran 19839, Iran
}

28 March 2022

\begin{abstract}
We examine linear perturbation theory to evaluate the contribution of viscosity coefficient in the growing of dark matter perturbations in the context of the bulk viscous dark energy model inspired by thermodynamical dissipative phenomena proposed by Mostaghel et al. (2017). As the cosmological implementations, we investigate the Integrated Sachs-Wolfe (ISW) auto-power spectrum, the ISW-galaxy cross-power spectrum and derive limits on $f \sigma_{8}$. The dimensionless bulk viscosity coefficient $(\gamma)$ in the absence of interaction between dark sectors, modifies the Hubble parameter and the growth function, while the Poisson equation remains unchanged. Increasing $\gamma$ reduces the dark matter growing mode at the early epoch while a considerable enhancement will be achieved at the late time. This behavior imposes non-monotonic variation in the time evolution of gravitational potential generating a unique signature on the CMB photons. The bulk viscous dark energy model leads to almost a decreasing in ISW source function at the late time. Implementation of the Redshift Space Distortion (RSD) observations based on "Gold-2017" catalogue, shows $\Omega_{\mathrm{m}}^{0}=0.303_{-0.038}^{+0.044}$, $\gamma=0.033_{-0.033}^{+0.098}$ and $\sigma_{8}=0.769_{-0.089}^{+0.080}$ at $1 \sigma$ level of confidence. Finally, tension in the $\sigma_{8}$ is alleviated in our viscous dark energy model.
\end{abstract}

Key words: methods: analytical-methods: data analysis - cosmic background radiation - dark energy - large-scale structure of Universe.

\section{INTRODUCTION}

The agent of the late time accelerating expansion of the Universe confirmed by many observational data sets ranging from background evolution to perturbations dynamics is mysterious not only for theoretical cosmology but also in observations (Riess et al. 1998; Perlmutter et al. 1999).

The standard cosmological model $(\Lambda \mathrm{CDM})$ containing six free parameters is a trivial prescription to explain dynamics of our Universe. This scenario has been confirmed by various observations such as Supernova Type Ia (SNIa), Cosmic Microwave Background radiation (CMB), Baryonic Acoustic Oscillations (BAO) (Ade et al. 2016a,b), the ISW effect (Boughn \& Crittenden 2004, 2005b,a; Ade et al. 2016c) and weak lensing (Peel et al. 2017; Heymans et al. 2013; Lewis \& Challinor 2006; Contaldi et al. 2003). Despite the outstanding consistencies between $\Lambda$ CDM and the observational cosmic data sets, the cosmological constant has some fundamental problems and concordance model has tensions remained unresolved (Ade et al. 2016a;

* email:m.s.movahed@ipm.ir
Riess et al. 2016; Bernal et al. 2016). Recent observations indicated a deficiency in amplitude of ISW cross-correlation with astronomical objects based on concordance model (Granett et al. 2008; Kovács et al. 2013; Flender et al. 2013; Ferraro et al. 2015). Taking into account the late-Universe measurements of the dark matter growth rate which is proportional to the $\sigma_{8}$ implied an orientation to low value with respect to that of computed by $\mathrm{CMB}$ in the context of $\Lambda$ CDM (Ade et al. 2016c; Heymans et al. 2012; Erben et al. 2013). Subsequently, there is some room for alternative scenarios mainly classified into the following categories: Dynamical dark energy including the field theory orientation, phenomenological dark fluids, modification of the general relativity and thermodynamics point of view (Copeland et al. 2006; Amendola et al. 2013; Horndeski 1974; Könnig et al. 2016; Bento et al. 2002; Zlatev et al. 1999; Caldwell 2002; Amendola 1993; Germani \& Kehagias 2010; Huterer \& Shafer 2018; Mostaghel et al. 2017, and references therein). In an interesting approach, recently, N. Khosravi suggested a new proposal to modify the standard cosmology based on the idea of taking ensemble average over the various gravitational models (Khosravi et al. 
2017; Khosravi 2018, 2016). The $\Lambda(t)$ cosmology, considering a typical form of dark energy and/or interaction between dark sectors in the Universe are some proposals to reduce such discrepancies (Wang et al. 2010a; Velten et al. 2015; Kunz et al. 2015; Barbosa et al. 2017; Fan et al. 2016; Mainini \& Mota 2011).

Another proposal for dark energy is an exotic fluid with some thermodynamical features such as bulk and/or shear viscosities. Meanwhile, finding proper observational measures which can precisely probe the influence of dark energy component and distinguishing between various scenarios have received extensive attention. Geometrical and topological properties of cosmological random fields (Ling et al. 2015; Fang et al. 2017) and considering the primary and the secondary probes have been discovered and applied for evaluation and discrimination of dark energy models (Huterer \& Shafer 2018, and references therein).

Dark energy can affect on the various elements of the Universe. A trivial contribution of dark energy can be realized in the rate of background expansion. Hence all quantities containing the Hubble parameter are affected by dark energy density. At the background level, due to changing the expansion history of the Universe, the distance to the last scattering surface is modified changing the so-called acoustic signatures ( $\mathrm{Hu} \&$ White 1996). Dark energy perturbations can also alter the lensing potential (Acquaviva \& Baccigalupi 2006; Carbone et al. 2013) and consequently can modify the lensing $B$-mode (Amendola et al. 2014). Dark energy modifies the primordial gravitational wave and therefore it changes the amplitude of primordial B-mode (Antolini et al. 2013; Raveri et al. 2015; Amendola et al. 2014). The matter perturbations growth is also affected by dark energy density (Peebles 1984; Barrow \& Saich 1993) causing to have some discrepancies between amplitude of fluctuations computed by late-time observations and CMB map (Durrer et al. 1999; Baldi \& Pettorino 2011; Ade et al. 2016b).

Secondary CMB anisotropies are widely experiencing different epoch of the Universe, therefore, we expect that the CMB stochastic field is a proper measure to explore dark energy. A relevant probe of dark energy in the context of CMB observations, is ISW effect which is a secondary anisotropy (Sachs \& Wolfe 1967; Rees \& Sciama 1968; Kofman \& Starobinskij 1985; Hu \& Sugiyama 1994; Crittenden \& Turok 1996; Cooray 2002; Afshordi 2004; Schäfer \& Bartelmann 2006; Schäfer 2008b; Ade et al. 2016c). ISW effect is related to the frequency changes in the CMB photons when they encounter with the time evolving gravitational potential. Since dark energy is dominating at the low redshift ${ }^{1}(z \sim 1)$, the primordial CMB fluctuations alone cannot provide a considerable and precise probe. However, the secondary anisotropies in the CMB is more sensitive to the dark energy dynamics (Schäfer 2008b; Kovács et al. 2013; Huterer \& Shafer 2018). The cosmic variance for almost those multipoles that ISW has signature on the $\mathrm{CMB}$ power spectrum washouts the

1 It turns out that various dark energy cosmological models can provide different range of redshifts depending on corresponding natures. importance of ISW alone to explore dark energy models (Song et al. 2007).

Practically, the cross-correlation of ISW with the tracers of the large scale structures magnifies the ISW signal and it would be distinguishable from primordial processes (Afshordi et al. 2004; Afshordi 2004; Ho et al. 2008; Olivares et al. 2008; Giannantonio et al. 2008; Douspis et al. 2008; Wang et al. 2010a; Ferraro et al. 2015; Lesgourges 2013).

The ISW signal has been computed, in order to examine the $\Lambda$ contribution in concordance model relying on rare superstructures identified in the SDSS Luminous Red Galaxy catalogue (Nadathur et al. 2012; Flender et al. 2013). The ISW effect and its cross-correlation with large scale structures have been investigated in the context of alternative to $\Lambda$ CDM models such as the extended quintessence model in both the metric and Palatini formalisms (Fan et al. 2016), quintessence cosmological model (Wang et al. 2010b), particular form of interaction between dark sectors (Schäfer 2008b; Schäfer 2008a; Schäfer et al. 2009), non-ideal fluid dark energy with anisotropic stress component (Majerotto et al. 2015), clustering of dark energy (Khosravi et al. 2016) and also other more general cosmological models (Scranton et al. 2003; Ferraro et al. 2015; Velten et al. 2015; Fosalba et al. 2003; Dent et al. 2009; Sapone \& Kunz 2009; Padmanabhan et al. 2005; Dent et al. 2009).

More recently, inspired by thermodynamical dissipative phenomena and taking into account the isotropy of the Universe at the background level, we proposed a bulk viscous dark energy (BVDE) model (Mostaghel et al. 2017). In this model, the old cosmological objects can be accommodated and the tension in the Hubble parameter was alleviated (Mostaghel et al. 2017). Relying on dissipative process in a realistic fluid, Israel et al. proposed a causal dissipative theory for assessing the irreversible processes (Israel \& Stewart 1979). Accordingly, bulk and shear viscous terms are most relevant parts for a feasible relativistic fluid. The Ekart's theory including the first-order dissipative relativistic fluid, is acausal and has instabilities (Eckart 1940; Hiscock \& Lindblom 1985; Hiscock \& Salmonson 1991) (see also (Landau \& Lifshitz 1987; Jou et al. 2009)). There are many approaches to construct causal and stable theory of relativistic viscous fluid for a certain range of relevant quantities and proper conditions (Hiscock \& Lindblom 1983, 1988; Disconzi 2014). However, examining the problem of causality and stability of relativistic theories is under debate (Rezzolla \& Zanotti 2013).

For cosmological implementation, it has been demonstrated that Israel approach converges to the Eckart's theory (Hiscock \& Salmonson 1991). Since, the collision time scale in the transport equation of our proposed fluid is zero, consequently, our bulk viscous model is necessarily acausal and unstable (Maartens 1996). For making a causal bulk viscous dark energy, in principle, one should take into account full Israel-Stewart transport equation and keeping the collision time scale (Israel \& Stewart 1979). On the other hand, the functional form of viscosity in our model leads to crossing phantom divide barrier which has been observationally confirmed (Ade et al. 2016b). In order to resolve mentioned instability and regularize underlying model, in some cases, 
one can take into account interaction between dark components of the Universe (Amendola et al. 2013). The effective dark energy in a suitable interacting model can possibly have phantom crossing without divergences and it is fundamentally related to the collision time scale in the corresponding transport equation. It is worth noting that in a simple dark energy model with constant equation of state accompanying a typical interaction term leads to another instability (Väliviita et al. 2008). One approach to reduce the effect of instability may possibly be assumed a proper rate of interaction between viscous dark energy and dark matter. Another way for regularization the instability is suppressing any initial perturbations of dark energy by taking proper initial conditions.

In this paper, we are interested in discussing the consequence of BVDE model incorporating the background expansion on the large scale structures. To this end, we will take into account the linear perturbation of dark matter in the presence of our viscous dark energy model to study its contributions on the rate of structure formations and ISW effect ${ }^{2}$.

We will focus on the ISW auto-power spectrum and cross-correlation between CMB fluctuations mainly considered by ISW part with large scale structure to explore the contribution of bulk viscosity in the dark energy budget. To make our discussion more complete, we will use the Redshift Space Distortion (RSD) data set to constrain model free parameters. The contribution of viscosity coefficient represents a non-monotonic behavior of ISW auto- and cross-power spectrums leading to low clustering compared to $\Lambda \mathrm{CDM}$ model and also it can reduce the tension in $\sigma_{8}$. It is worth reviewing that the BVDE cosmological model could alleviate $H_{0}$ tension (Mostaghel et al. 2017).

The layout of the rest of this paper is as follows: In section 2, for the sake of clarity, we give a brief introduction of our bulk viscous dark energy (BVDE) model. Background dynamics of the Universe will be explained in this section. Section 3 is devoted to the linear perturbation of the dark matter when bulk viscous dark energy is considered. Growth function, growth rate and bias independent parameter, namely $f \sigma_{8}$ for BVDE model are derived in section 3. Angular auto-power spectrum of ISW and ISW-galaxy power spectrum in the flat sky approximation for BVDE model will be derived in section 4 . We will check the observational consistency using RSD data set in section 5. Summary and concluding remarks are given in section 6 .

\section{COSMIC EVOLUTION IN THE BULK VISCOUS DARK ENERGY FRAMEWORK}

A proposal for dark energy model inspired by dissipative fluid dynamics is considering bulk and shear viscous terms for the energy-momentum tensor. Recently, we consider an exotic dissipative fluid playing a responsible for the late time

2 Since there are various types of bulk viscosity models for dark energy cosmologies, some authors have tried to evaluate linear structure formation process (Barbosa et al. 2017, and references therein). acceleration (Mostaghel et al. 2017). The pressure for dark energy (DE) component in the BVDE model is defined by:

$$
p_{\mathrm{DE}}=-\rho_{\mathrm{DE}}-\zeta \Theta(t),
$$

where $\zeta=\zeta\left(\rho_{\mathrm{DE}}\right)$ and $\Theta(t)=\nabla^{\mu} u_{\mu}$ are viscosity and expansion scalar, respectively. In the FLRW space-time, expansion scalar is $\Theta(t)=3 H(t)=3 \dot{a} / a$. In addition, we have an assumption (Mostaghel et al. 2017):

$$
\zeta\left(\rho_{\mathrm{DE}}, H\right)=\xi \frac{\sqrt{\rho_{\mathrm{DE}}}}{H},
$$

where $\xi$ is positive constant with mass square dimension. Other functional form for $\zeta$ can be found in ( $\mathrm{Li} \&$ Barrow 2009; Hiscock \& Salmonson 1991; Barbosa et al. 2017, and references therein). By solving the continuity equation, the evolution of dark energy component becomes:

$$
\rho_{\mathrm{DE}}(a)=\rho_{\mathrm{DE}}^{0}\left(1+\frac{9 \xi}{2 \sqrt{\rho_{\mathrm{DE}}^{0}}} \ln a\right)^{2} .
$$

The superscript "0" represents the value of DE component at present time. Using the flat FLRW metric in the Einstein's fields equations, we find the Friedmann equations as:

$H^{2}=\frac{8 \pi \mathrm{G}_{\mathrm{N}}}{3} \rho_{\mathrm{tot}}$,
$\frac{\ddot{a}}{a}=-\frac{4 \pi \mathrm{G}_{\mathrm{N}}}{3}\left(\rho_{\mathrm{tot}}+3 p_{\mathrm{tot}}\right)$,

where $\rho_{\text {tot }}=\rho_{\mathrm{r}}+\rho_{\mathrm{b}}+\rho_{\mathrm{m}}+\rho_{\mathrm{DE}}$ and $p_{\text {tot }}=p_{\mathrm{r}}+p_{\mathrm{b}}+p_{\mathrm{m}}+$ $p_{\text {DE }}$. The $G_{N}$ is Newton's constant. The indices "r", "b" and "m" correspond to radiation, baryonic matter and cold dark matter (CDM), respectively. Finally, the Hubble parameter without any interaction between dark sectors reads:

$$
\begin{aligned}
H^{2}= & H_{0}^{2}\left[\Omega_{\mathrm{r}}^{0} a^{-4}+\Omega_{\mathrm{m}}^{0} a^{-3}+\Omega_{\mathrm{DE}}^{0}\left(1+\frac{9 \gamma}{2 \sqrt{\Omega_{\mathrm{DE}}^{0}}} \ln a\right)^{2}\right] \\
& +H_{0}^{2}\left(1-\Omega_{\mathrm{tot}}^{0}\right) a^{-2}
\end{aligned}
$$

where $\Omega_{\mathrm{tot}}^{0}=\Omega_{\mathrm{r}}^{0}+\Omega_{\mathrm{m}}^{0}+\Omega_{\mathrm{DE}}^{0}$ and throughout this paper, we consider flat Universe $\left(\Omega_{\mathrm{tot}}=1\right)$. In Eq. (5), we define:

$\Omega_{\mathrm{r}}^{0} \equiv \frac{8 \pi \mathrm{G}_{\mathrm{N}}}{3 H_{0}^{2}} \rho_{\mathrm{r}}^{0}, \quad \Omega_{\mathrm{m}}^{0} \equiv \frac{8 \pi \mathrm{G}_{\mathrm{N}}}{3 H_{0}^{2}} \rho_{\mathrm{m}}^{0}, \quad \Omega_{\mathrm{DE}}^{0} \equiv \frac{8 \pi \mathrm{G}_{\mathrm{N}}}{3 H_{0}^{2}} \rho_{\mathrm{DE}}^{0}$,

and the dimensionless viscosity coefficient, $\gamma$ is:

$$
\gamma \equiv \sqrt{\frac{8 \pi \mathrm{G}_{\mathrm{N}}}{3 H_{0}^{2}}} \xi .
$$

It turns out that for $\gamma=0$, the standard $\Lambda$ CDM model is recovered. Due to viscosity term, we have non-trivial behavior for DE in BVDE model compared to cosmological constant. In Fig. 1, we indicate the evolution of $\Omega_{\mathrm{DE}}(a)$ as a function of scale factor. As indicated in the inset plot of this figure, around $a \lesssim 0.06$ the contribution of DE in the BVDE model is more than $\Lambda$. While for the interval $0.06 \lesssim a \lesssim 0.9$ the value of $\Omega_{\mathrm{DE}}$ is less than $\Lambda \mathrm{CDM}$ model. At very early epoch, again the $\Omega_{\mathrm{DE}}$ is similar to $\Lambda$. Subsequently, this kind of behavior has non-trivial impact on the ISW as well as other structure formation phenomena. In the next section, we will study the structure formation in the BVDE framework. 


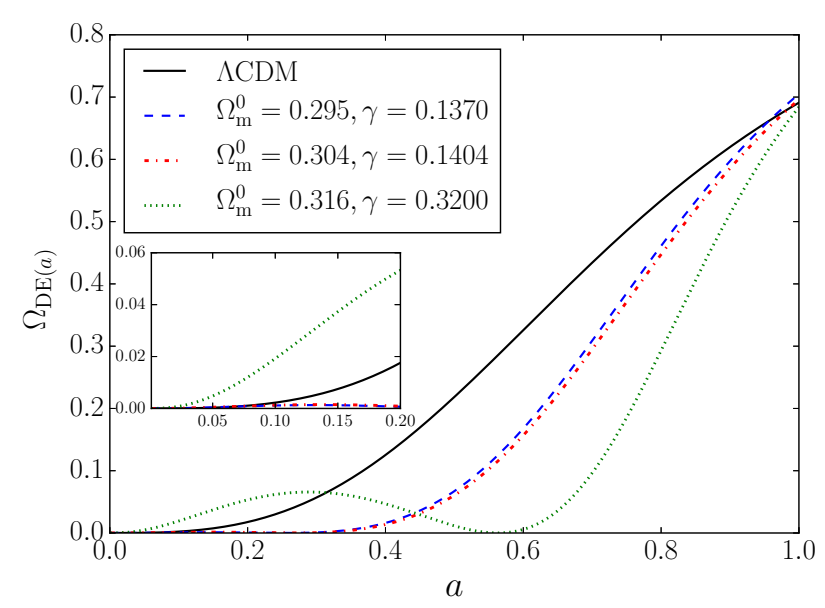

Figure 1. The energy density of dark energy in the $\Lambda$ CDM and BVDE models as a function of scale factor. The values of the free parameters selected in this plot are compatible with background tests (Mostaghel et al. 2017).

\section{STRUCTURE FORMATION IN THE BVDE MODEL}

Cosmic fluctuations seeded by inflationary models and/or topological phase transitions at the early Universe, are evolving through the aging of the Universe and finally form the large scale structures. In this section we explain the main part of structure formation and associated physical parameters such as dark matter power spectrum in the presence of BVDE model.

In order to study the evolution of the large scale structures in the Universe filled by cold dark matter (CDM) and viscous dark energy, we consider Einstein equations for small inhomogeneities as:

$\delta G_{\nu}^{\mu}=8 \pi \mathrm{G}_{\mathrm{N}} \delta \mathrm{T}_{\nu}^{\mu}$.

The perturbed metric in the Newtonian gauge for the homogeneous and isotropic Universe is therefore written by (Bardeen (1980); Kodama \& Sasaki (1984); Mukhanov (1992)):

$\mathrm{d} s^{2}=a^{2}(\eta)\left[-(1+2 \Psi) \mathrm{d} \eta^{2}+(1+2 \Phi) \delta_{i j} \mathrm{~d} x^{i} \mathrm{~d} x^{j}\right]$,

where $\eta, \Psi$ and $\Phi$ are conformal time and metric perturbations, respectively. In absence of anisotropic stress, we have $\Psi=-\Phi$. Applying the above line element in the Einstein's equations leads to the perturbed gravitational field equations (Bardeen 1980; Kodama \& Sasaki 1984; Mukhanov 1992):

$\delta G_{0}^{0}=2 a^{-2}\left[3 \mathcal{H}\left(\mathcal{H} \Psi-\Phi^{\prime}\right)+\square \Phi\right]$,

$\delta G_{i}^{0}=2 a^{-2}\left(\Phi^{\prime}-\mathcal{H} \Psi\right)_{\mid i}$,

$\delta G_{j}^{i}=2 a^{-2}\left[\left(\mathcal{H}^{2}+2 \mathcal{H}^{\prime}\right) \Psi+\mathcal{H} \Psi^{\prime}-\Phi^{\prime \prime}-2 \mathcal{H} \Phi^{\prime}\right] \delta_{i j}$

$$
+a^{-2}\left[\nabla^{2}(\Psi+\Phi) \delta_{j}^{i}-(\Psi+\Phi)_{\left.\right|_{j} ^{i}}\right]
$$

The conformal Hubble parameter is $\mathcal{H}=a^{\prime} / a$, where prime denotes the derivative with respect to the conformal time $\eta$. In the Eqs. (10), subscript "|" is the covariant derivative with respect to the spatial 3-dimensional metric and the box operator is $\square=\nabla^{\mu} \nabla_{\mu}$. The linear perturbation of CDM energy momentum tensor becomes $\delta T_{\nu}^{\mu}=\rho_{\mathrm{m}}\left[\Delta_{\mathrm{m}} u_{\nu} u^{\mu}+\right.$ $\left.u^{\mu} \delta u_{\nu}+u_{\nu} \delta u^{\mu}\right]$ and density contrast is represented by $\Delta_{\mathrm{m}} \equiv$ $\delta \rho_{\mathrm{m}} / \rho_{\mathrm{m}}$. By applying Fourier Transformation of the perturbation equations, we find the following perturbed Einstein equations:

$k^{2} \tilde{\Phi}+3 \mathcal{H}\left(\tilde{\Phi}^{\prime}-\mathcal{H} \tilde{\Psi}\right)=4 \pi \mathrm{G}_{\mathrm{N}} \mathrm{a}^{2} \tilde{\Delta}_{\mathrm{m}} \rho_{\mathrm{m}}$,

$k^{2}\left(\tilde{\Phi}^{\prime}-\mathcal{H} \tilde{\Psi}\right)=-4 \pi \mathrm{G}_{\mathrm{N}} \mathrm{a}^{2} \rho_{\mathrm{m}} \tilde{\theta}$,

$\tilde{\Psi}=-\tilde{\Phi}$,

$\tilde{\Phi}^{\prime \prime}+2 \mathcal{H} \tilde{\Phi}^{\prime}-\mathcal{H} \tilde{\Psi}^{\prime}-\left(\mathcal{H}^{2}+2 \mathcal{H}^{\prime}\right) \tilde{\Psi}=0$.

The sign " " corresponds to the Fourier mode. The velocity divergence in the Eqs. (11) is $\tilde{\theta} \equiv \mathbf{i} k_{j} \tilde{v}^{j}$. Combining Eqs. (11) and continuity equation, the evolution equation for the CDM density contrast at the linear regime on the sub-horizon scales is as follows:

$\frac{\mathrm{d}^{2} \tilde{\Delta}_{\mathrm{m}}}{\mathrm{d} N^{2}}+\left(\frac{\mathrm{d} \ln \mathcal{H}}{\mathrm{d} N}+1\right) \frac{\mathrm{d} \tilde{\Delta}_{\mathrm{m}}}{\mathrm{d} N}-\frac{3}{2} \tilde{\Delta}_{\mathrm{m}}=0$,

where $N \equiv \ln a$ is the number of $e$-foldings. One can derive a set of differential equations representing the perturbations in the BVDE component. However, we consider suitable initial conditions to suppress the dark energy perturbations and finally, the effect of instability is resolved.

Since Eq. (12) is independent from scale of structure, therefore, one can define $\tilde{\Delta}_{\mathrm{m}}(\mathbf{k}, a) \equiv \delta_{\mathrm{m}}(a) \tilde{\Delta}_{\mathrm{m}}(\mathbf{k})$ with two independent modes which are called decaying $\left(\delta_{\mathrm{m}}^{-}\right)$and growing $\left(\delta_{\mathrm{m}}^{+}\right)$modes. The linear growth rate of the density contrast, $f$, which is related to the peculiar velocity in the linear theory is defined by (Peebles 1993):

$$
f(a) \equiv \frac{\mathrm{d} \ln D_{\mathrm{m}}^{+}(a)}{\mathrm{d} \ln a}
$$

where $D_{\mathrm{m}}^{+}(a) \equiv \delta_{\mathrm{m}}^{+}(a) / \delta_{\mathrm{m}}^{+}(a=1)$ which is known as growth function. The growth rate measurements are characterized by the peculiar velocities obtained from the Redshift Space Distortion (RSD) observations (Kaiser 1987). To achieve proper observational quantity comparable with the growth rate computed from the linear perturbation theory, one can compare transverse and line of sight anisotropies influenced by the peculiar motion in the redshift space clustering of galaxies. Weak lensing and/or RSD (Song \& Percival 2009; Nesseris et al. 2017) yield a robust combination, namely $f \sigma_{8}(z) \equiv f(z) \sigma_{8}(z)$ which is bias independent observable quantity. Here the variance of the linear density contrast on scale $R_{8}=8 h^{-1} \mathrm{Mpc}$ is $\sigma_{8}(z) \equiv \sigma\left(R_{8}, z\right)$ and it is given by:

$\sigma\left(R_{8}, z\right)=D_{\mathrm{m}}^{+}(z)\left[\int_{0}^{\infty} \frac{\mathrm{d} k}{2 \pi^{2}} k^{2} \mathcal{P}_{\mathrm{m}}(k) W^{2}\left(k R_{8}\right)\right]^{1 / 2}$

where $W(k R)=\frac{3(\sin k R-k R \cos k R)}{(k R)^{3}}$ and $R=\left(3 M / 4 \pi \rho_{\mathrm{m}}\right)^{1 / 3}$. The matter power spectrum is introduced by $\left\langle\tilde{\Delta}_{\mathrm{m}}(\mathbf{k}) \tilde{\Delta}_{\mathrm{m}}\left(\mathbf{k}^{\prime}\right)\right\rangle=(2 \pi)^{3} \delta_{\mathrm{D}}\left(\mathbf{k}-\mathbf{k}^{\prime}\right) \mathcal{P}_{\mathrm{m}}(\mathrm{k})$. Theoretical formula for the present matter power spectrum is $\mathcal{P}_{\mathrm{m}}(k)=\mathcal{P}_{0} k^{n_{s}} T^{2}(k)$ where $\mathcal{P}_{0}$ is normalization constant and $n_{s}=0.968 \pm 0.006$ according to the recent reanalysis of the Planck data (Ade et al. 2016a). $T(k)$ is transfer function. Since, at the very high redshift, our BVDE model is almost similar to that of supposed by $\Lambda \mathrm{CDM}$, therefore, we use the BBKS transfer function model (Bardeen et al. 1986):

$T(k)=C_{q}\left[1+3.89 q+(16.1 q)^{2}+(5.46 q)^{3}+(6.71 q)^{4}\right]^{-1 / 4}$, 


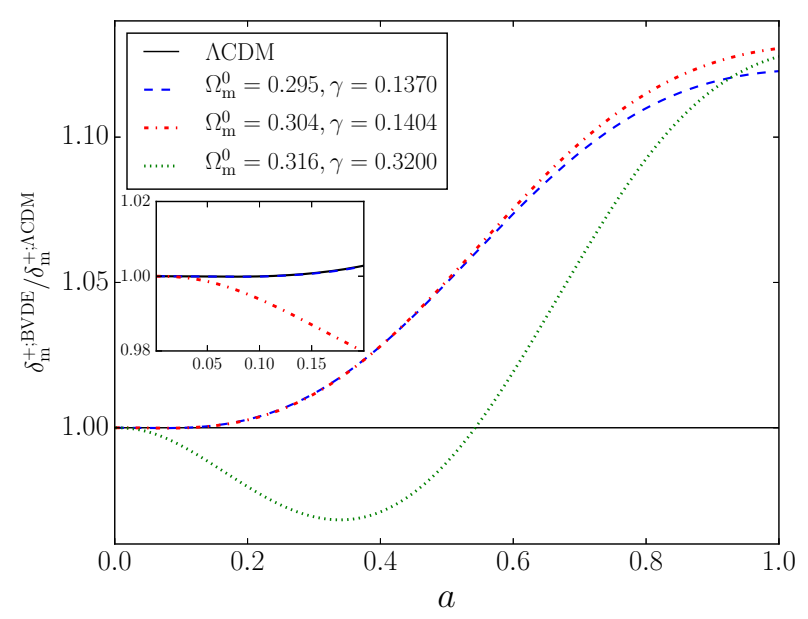

Figure 2. The growing mode ratio of BVDE and $\Lambda$ CDM, $\delta_{\mathrm{m}}^{+; \mathrm{BVDE}} / \delta_{\mathrm{m}}^{+; \Lambda \mathrm{CDM}}$ for different values of free parameters as a function of scale factor. The value of free parameters obtained from the background estimations reported by Ref. Mostaghel et al. (2017).

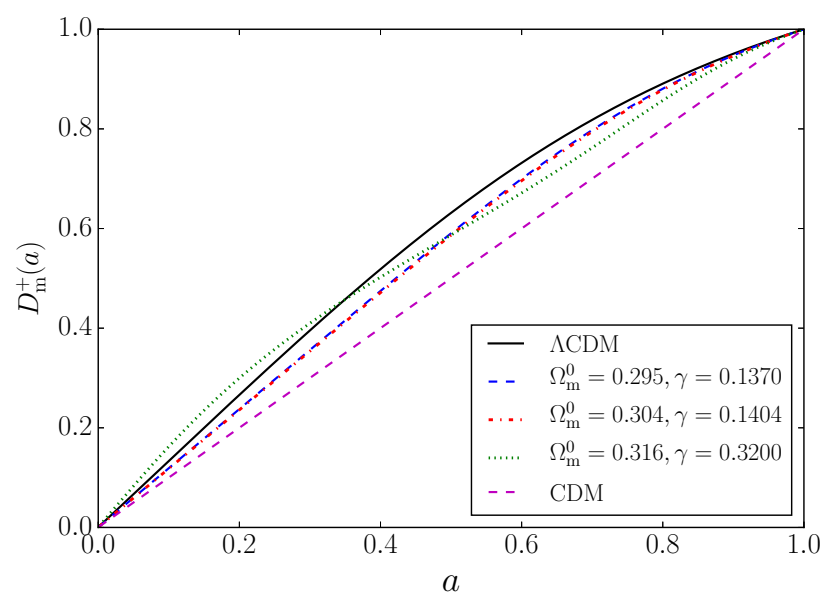

Figure 3. Evolution of CDM growth function as a function of scale factor for various values of free parameters. These values have been set according to background estimations represented in the Ref. Mostaghel et al. (2017).

where $C_{q} \equiv \ln (1+2.34 q) / 2.34 q$ and $q \equiv k / \Gamma$. Here $\Gamma$ is the shape parameter, given by:

$\Gamma=\Omega_{\mathrm{m}}^{0} \tilde{h} \exp \left(-\Omega_{\mathrm{b}}^{0}-\sqrt{2 \tilde{h}} \Omega_{\mathrm{b}}^{0} / \Omega_{\mathrm{m}}^{0}\right)$.

It is worth noting that in the general case, one should recalculate the transfer function to find robust results (Wang et al. 2010b, and references therein) (e.g. to assess non-linear evolution see (Smith et al. 2003; McDonald et al. 2006; Lewis \& Challinor 2006)).

Fig. 2 indicates the ratio of BVDE growing mode to the $\Lambda \mathrm{CDM}$ model, i.e. $\delta_{\mathrm{m}}^{+; \mathrm{BVDE}} / \delta_{\mathrm{m}}^{+; \Lambda \mathrm{CDM}}$. Our result shows that at the early epoch, growing mode in the BVDE model is smaller than that of in $\Lambda \mathrm{CDM}$, on the contrary, at the late time, this quantity becomes higher than growing mode in $\Lambda \mathrm{CDM}$. However, incorporating bulk viscosity for the dark

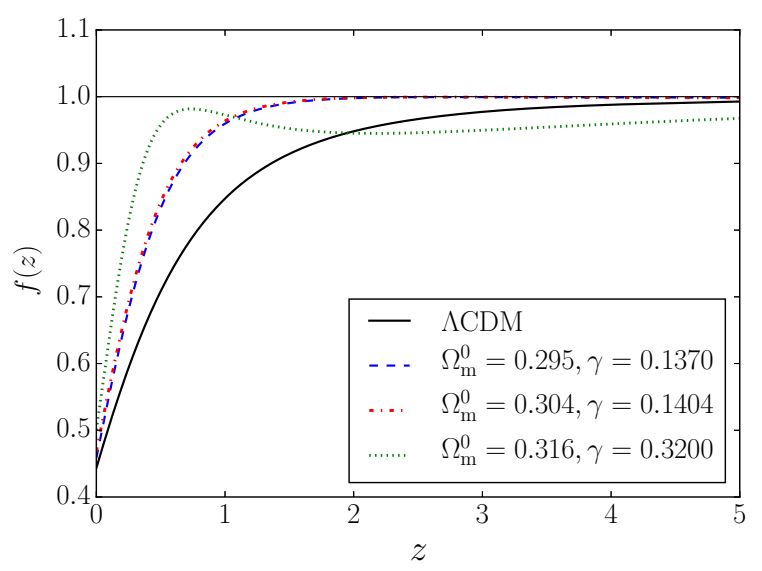

Figure 4. Growth rate of model for different values of viscosity in comparison of $\Lambda \mathrm{CDM}$.

energy fluid leads to an early dark energy (see Fig. 1). Such component for the dark energy with negative pressure, can reduce the rate of large scale structure formation during the matter dominant Universe. As illustrated in Fig. 2, the growing mode is not monotonic function versus scale factor and structure formation experiences a delay in the presence of viscous dark energy.

In Fig. 3, we plot the evolution of $D_{\mathrm{m}}^{+}(a)$, as a function of scale factor. By increasing the bulk viscosity coefficient in the BVDE model, we find a deviation from the standard $\Lambda$ CDM model. Subsequently, the BVDE cosmological model predicts a different rate of structure formation, leading to a new observational consequences at relevant redshifts.

We also depict the linear growth rate in Fig. 4. For almost $\gamma \lesssim \gamma_{\times} \sim 0.36$ (Mostaghel et al. 2017), the early dark energy contribution can not compensate the higher role of cold dark matter at the late time. Consequently, the growth rate is higher than $\Lambda$ CDM. Such behavior is no longer valid for almost $\gamma \gtrsim \gamma_{\times}$as illustrated in Fig. 4. There are more abundance of CDM clusters around $z \sim 0.8$ for higher value of viscosity coefficient. This behavior is due to the slowly varying scale factor while the perturbations have opportunity to grow up (Lahav et al. 1991). We also expect that for higher value of viscosity coefficient, the CMB photons have more chance to experience dynamical gravitational potential at the intermediate scale factor (Fig. 1). In the next section, we will examine the ISW contribution on the CMB map due to the BVDE model, precisely.

\section{INTEGRATED SACHS-WOLFE EFFECT}

One of the sensitive and feasible tracers of the dark energy density is ISW effects. This effect is due to the interaction of $\mathrm{CMB}$ photons with time-varying gravitational potential. This term contains all processes due to the non-static metric fluctuations. During radiation and dark energy dominated epochs, the gravitational potential, $\Phi$, has dynamics leading to non-vanishing contribution on the CMB fluctuations. Here, we ignore the non-significant early ISW effect associated with the radiation dominated Uni- 
verse. The quantitative formula for computing the ISW contribution on the CMB fluctuation seen in direction $\hat{\mathbf{n}}$ reads as (Sachs \& Wolfe 1967; Seljak \& Zaldarriaga 1996; Gordon \& Hu 2004; Afshordi et al. 2004; Ho et al. 2008; Olivares et al. 2008; Ade et al. 2016c):

$$
\left(\frac{\Delta T}{T_{\mathrm{CMB}}}\right)(\hat{\mathbf{n}})=-2 \int_{0}^{\chi_{\mathrm{CMB}}} \mathrm{d} \chi a^{2} H(a) \frac{\partial \Phi(\hat{\mathbf{n}}, a)}{\partial a},
$$

here $\chi_{\mathrm{CMB}}$ is the comoving distance to the last scattering surface. Using the Eq. (11a), the Poisson equation in the Fourier space for the scales smaller than the Hubble radius, $k \gg \mathcal{H}$, becomes:

$k^{2} \tilde{\Phi}=4 \pi \mathrm{G}_{\mathrm{N}} \mathrm{a}^{2} \rho_{\mathrm{m}} \delta_{\mathrm{m}}^{+}(\mathrm{a}) \tilde{\Delta}_{\mathrm{m}}(\mathbf{k})$

Finally, we get the gravitational potential as:

$\tilde{\Phi}=\frac{3 H_{0}^{2}}{2 k^{2}} a^{2} E^{2}(a) \Omega_{\mathrm{m}}(a) \delta_{\mathrm{m}}^{+}(a) \tilde{\Delta}_{\mathrm{m}}(\mathbf{k})$,

where $E^{2}(a) \equiv H^{2}(a) / H_{0}^{2}$. Now we define the function $Q(a)$ by (Schäfer 2008b):

$$
Q(a) \equiv a^{2} E^{2}(a) \Omega_{\mathrm{m}}(a) D_{\mathrm{m}}^{+}(a) \delta_{\mathrm{m}}^{+}(a=1),
$$

Finally, the Eq. (17) equates to:

$$
\begin{aligned}
& \left(\frac{\Delta T}{T_{\mathrm{CMB}}}\right)(\hat{\mathbf{n}})= \\
& -3 H_{0}^{2} \int_{0}^{\chi_{\mathrm{CMB}}} \mathrm{d} \chi a^{2} H(a) \frac{\mathrm{d} Q(a)}{\mathrm{d} a} \int \frac{\mathrm{d} \mathbf{k}}{(2 \pi)^{3 / 2} \mathrm{k}^{2}} \mathrm{e}^{-\mathbf{i k} \cdot \hat{\mathbf{n}} \chi} \tilde{\Delta}_{\mathrm{m}}(\mathbf{k})
\end{aligned}
$$

According to this quantitative equation, the $Q(a)$ function and its derivative are the sources of ISW effect (Schäfer 2008b; Wang et al. 2010a,b). In our BVDE model, the ISW is altered by modified Hubble parameter and growth function, while the Poisson equation remains unchanged due to the absence of interaction between the BVDE and cold dark components. As indicated in the upper part of Fig. 5 , the $Q(a)$ function in the BVDE model is lower than the $\Lambda$ CDM model. The $\mathrm{d} Q(a) / \mathrm{d} a$ behaves as non-monotonic function versus scale factor leading to non-trivial contribution in the ISW effect. Actually taking into account the $\gamma$ (dimensionless viscosity coefficient), the magnitude of $\mathrm{d} Q(a) / \mathrm{d} a$ as a source of ISW, becomes smaller than $\Lambda$ CDM . This behavior can be justified by considering the contribution of $\Omega_{\mathrm{DE}}$ indicated in Fig. 1 and also by Fig. 3. In addition, the behavior of $H(a)$ in BVDE model is different compared to $\Lambda \mathrm{CDM}$ (Mostaghel et al. 2017). As discussed before, for $\gamma \gtrsim \gamma_{\times}$, we expect to have opposite contribution of BVDE resulting in the higher value for the source of ISW.

Till now we explained the physical model for ISW of CMB fluctuations, but due to stochasticity nature of $\mathrm{CMB}$ field, the practical observable measures are inferred from probabilistic framework in the context of n-point autocorrelation and cross-correlation approaches. We turn to the two-point correlation function of CMB temperature fluctuation expanded in terms of spherical harmonic basis functions: $C_{T}(\theta)=\sum_{\ell}(2 \ell+1) \mathcal{C}_{\ell}^{T T} \mathcal{W}_{\ell}^{2} P_{\ell}(\cos \theta) / 4 \pi$. Here $\mathcal{C}_{\ell}^{T T}$ is temperature angular power spectrum, $P_{\ell}$ is the Legendre polynomial and $\mathcal{W}_{\ell}$ is a smoothing function ${ }^{3}$. Considering

3 For a Gaussian kernel function, we have $\left.\mathcal{W}_{\ell}=\exp \left(-\theta_{\text {beam }}^{2} \ell(\ell+1) / 2\right)\right)$ and $\theta_{\text {beam }} \equiv \theta_{\mathrm{FWHM}} / \sqrt{8 \ln 2}$.

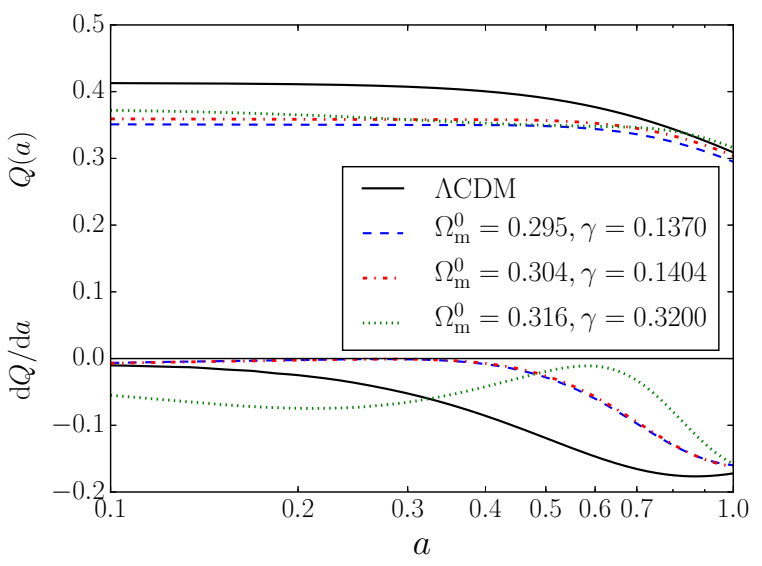

Figure 5. Upper part: The evolution of the $Q(a)$ function for various values of free parameters. Lower part: The evolution of the $\mathrm{d} Q(a) / \mathrm{d} a$ for the BVDE and $\Lambda$ CDM models. By definition $Q(a=1)=\Omega_{\mathrm{m}}^{0}$.

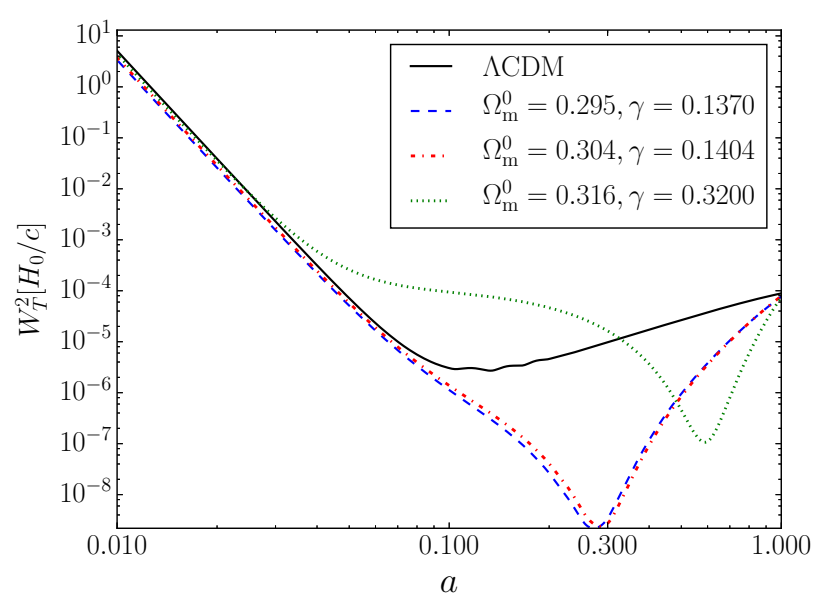

Figure 6. Weight function of ISW phenomenon versus scale factor for the BVDE and $\Lambda$ CDM models.

spherical harmonic expansion of $\Delta T / T_{\mathrm{CMB}}$ and taking into account Limber projection for simplification ${ }^{4}$ (Limber 1954; Kaiser 1992), ISW power spectrum reads as:

$$
\mathcal{C}_{T T, \ell}^{\mathrm{ISW}}=\int_{0}^{\chi_{\mathrm{CMB}}} \mathrm{d} \chi \mathcal{W}_{\ell}^{2} H_{0}^{4} \frac{W_{T}^{2}(\chi)}{\chi^{2}} \frac{\mathcal{P}_{\mathrm{m}}((\ell+1 / 2) / \chi)}{[(\ell+1 / 2) / \chi]^{4}},
$$

where $k=(\ell+1 / 2) / \chi$ (Ho et al. 2008) and the weighting function including the evolution of gravitational potential is $W_{T}(\chi) \equiv 3 a^{2} H(a) d Q(a) / d a$. Fig. 6 indicates $W_{T}^{2}$ as a function of scale factor for the BVDE and $\Lambda$ CDM models. For $\gamma \lesssim \gamma_{\times}$, the contribution of bulk viscosity decreases the amount of $W_{T}^{2}$ comparing to $\Lambda$ CDM . This is also justified by increasing the contribution of cold dark matter in the BVDE model. Non-monotonic behavior with respect to viscosity coefficient for $\gamma \gtrsim \gamma_{\times}$is a consequence of previous results shown in Fig. 5.

4 Actually, the integration over various values of $k$ is replaced by the most dominant contribution term. 


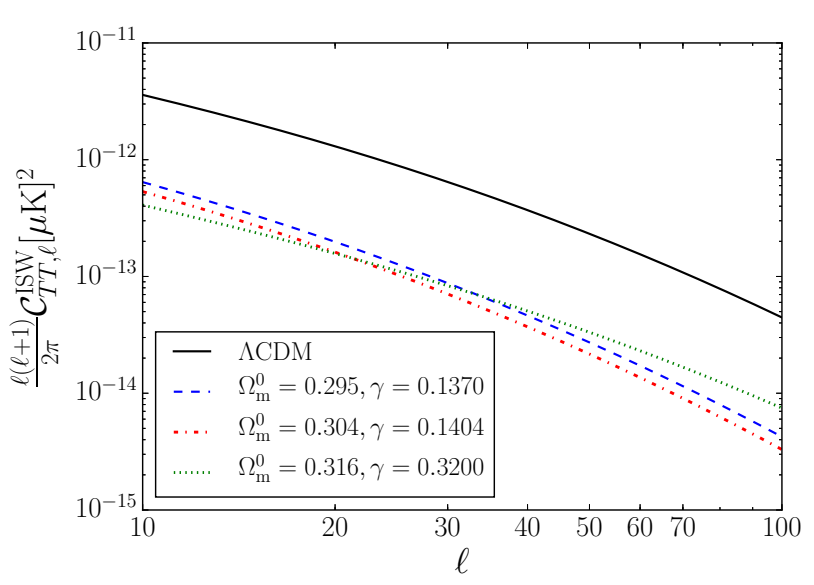

Figure 7. The ISW power spectrum $\mathcal{C}_{T T, \ell}^{\mathrm{ISW}}$ versus multiple order $\ell$ for the BVDE and $\Lambda$ CDM models.

Fig. 7 illustrates the effect of bulk viscosity on the ISW power spectrum. The ISW power spectrum for BVDE model is lower than $\Lambda \mathrm{CDM}$. Increasing $\gamma$ leads to decrease $\mathcal{C}_{T T, \ell}^{\mathrm{ISW}}$ for $\gamma \lesssim \gamma_{\times}$. This behavior is no longer valid for $\gamma \gtrsim \gamma_{\times}$due to the non-monotonic contribution of viscosity effect in the BVDE model.

Physical interpretation for this behavior is clarified by looking at the source terms in Eq. (22). For $\gamma \lesssim \gamma_{\times}$the ratio of $\Omega_{\mathrm{m}} / \Omega_{\mathrm{DE}}$ is higher than that of for $\Lambda \mathrm{CDM}$ during long period of evolution. Therefore, the amount of variation in the gravitational potential is less than $\Lambda$ CDM expressed by $\mathrm{d} Q(a) / \mathrm{d} a$ (a representative for $\dot{\Phi}$ ) in Fig. 5 resulting in the lower value of late ISW. For $\gamma \gtrsim \gamma_{\times}$, we expect to have more (less) contribution of viscous dark energy at the early (late) time. This manner leads to have a turning point for $\gamma$ dependency of ISW phenomenon in the BVDE model and CMB photons are experiencing more dynamical potential (see Fig. 5). The reducing in the $\Omega_{\mathrm{DE}} / \Omega_{\mathrm{m}}$ at the late time and in the $\mathrm{d} Q(a) / \mathrm{d} a$ can not be compensated by the more contribution of the viscous dark energy at the early time leading to have lower ISW with respect to $\Lambda \mathrm{CDM}$.

The physical processes governing on the CMB fluctuations can be classified into the primary and secondary classes. Therefore, the observed CMB map is a superposition of mentioned processes having almost different contributions in the various scales with almost same frequency dependent. Practically, it is very hard to distinguish the corresponding footprints. The early ISW effect which is due to the metric perturbation just after photon decoupling epoch on the sub-sound-horizon scales, has mainly contribution on the scale $\ell \sim 200$. On the other hand, late ISW due to evolving gravitational potential opportunity imprints on the small multipoles. Cosmic variance on small $\ell$ as well as the corresponding small value of amplitude are other obstacles for recognizing the ISW phenomenon alone (Wang et al. 2010a; Ferraro et al. 2015; Lesgourges 2013). A way to resolve this difficulty and constructing an observable power spectrum for ISW, is computing cross-correlation of observed CMB fluctuations with the tracers of the metric perturbations (Wang et al. 2010a,b, and references therein). This is because of spatial of ISW signal is correlated with the matter
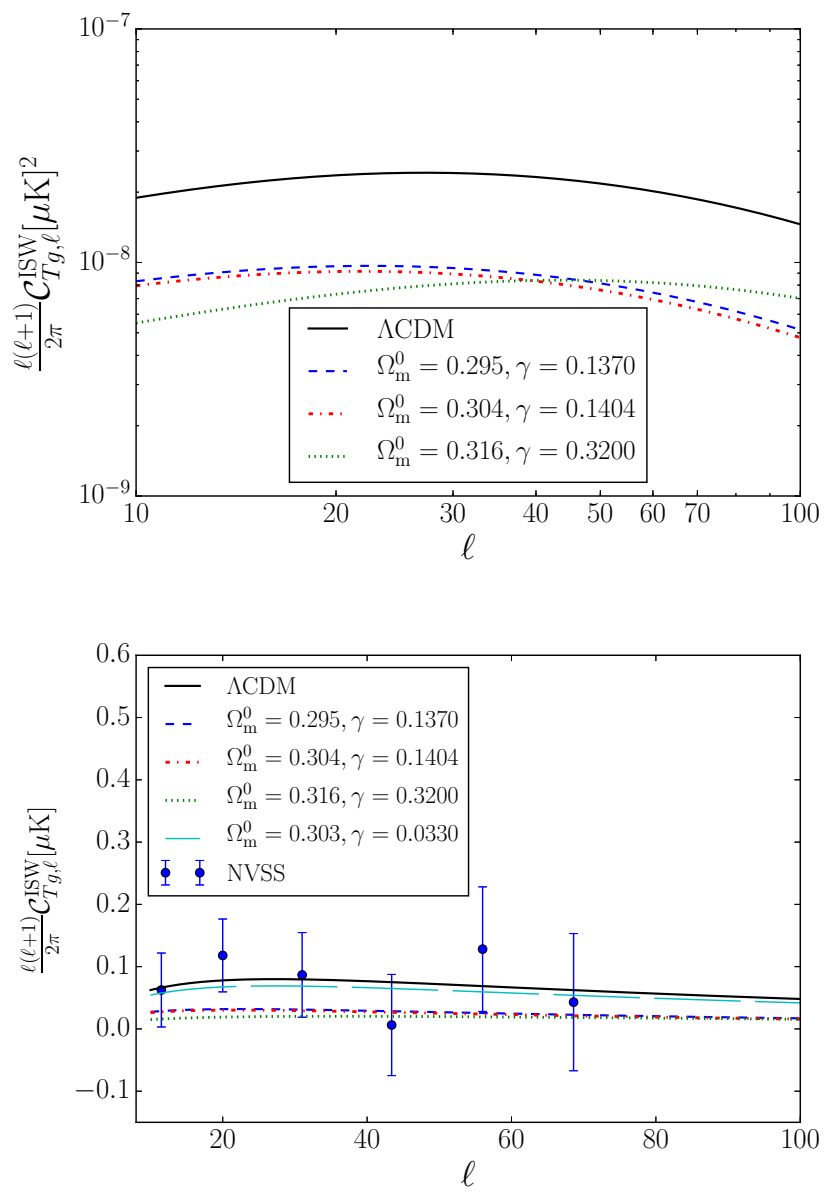

Figure 8. The ISW-cross power spectrum $\mathcal{C}_{T g, \ell}^{\mathrm{ISW}}$ versus multiple order $\ell$ for standard $\Lambda$ CDM and BVDE model. Here other cosmological parameters are fixed. The observed data points from NVSS surveys have been depicted.

distribution of the local Universe at low redshifts. To this end, we compute the cross-correlation of $\Delta T / T_{\mathrm{CMB}}$ with e.g. density contrast of galaxies or quasars, $\delta_{g}$. The scale independent density contrast of observable galaxies is:

$$
\delta_{g}(\hat{\mathbf{n}})=\int b(z) \frac{\mathrm{d} N}{\mathrm{~d} z} \Delta_{\mathrm{m}}(\hat{\mathbf{n}}, z) \mathrm{d} z
$$

where $b(z)$ is the bias between galaxies and dark matter density perturbations. Also, $\mathrm{d} N / \mathrm{d} z$ is the selection function of the survey. Similar to the previous strategy, the line of sight integral for density contrast of visible galaxies reads as:

$$
\begin{aligned}
\delta_{g}(\hat{\mathbf{n}})= & \int_{0}^{\chi_{\mathrm{CMB}}} \mathrm{d} \chi b(a) a^{2} \frac{\mathrm{d} N}{\mathrm{~d} a} H(a) D_{\mathrm{m}}^{+}(a) \delta_{\mathrm{m}}^{+}(a=1) \\
& \times \int \frac{\mathrm{d} \mathbf{k}}{(2 \pi)^{3 / 2}} \mathrm{e}^{-\mathrm{i} \mathbf{k} \cdot \hat{\mathbf{n}} \chi} \tilde{\Delta}_{\mathrm{m}}(\mathbf{k})
\end{aligned}
$$

We define the weight function for observable galaxy density contrast as: $W_{g}(\chi) \equiv b(a) a^{2}(\mathrm{~d} N / \mathrm{d} a) H(a) D_{\mathrm{m}}^{+}(a) \delta_{\mathrm{m}}^{+}(a=1)$. The angular cross-correlation of CMB fluctuations and visible galaxy density contrast considering only ISW contribu- 
tion in the flat-sky approximation is given by:

$\mathcal{C}_{T g, \ell}^{\mathrm{ISW}}=\int_{0}^{\chi_{\mathrm{CMB}}} \mathrm{d} \chi H_{0}^{2} \frac{W_{T}(\chi) W_{g}(\chi)}{\chi^{2}} \frac{\mathcal{P}_{\mathrm{m}}((\ell+1 / 2) / \chi)}{[(\ell+1 / 2) / \chi]^{2}}$,

In order to compute cross-correlation power spectrum, we use the NRAO VLA Sky Survey $\left(\right.$ NVSS) ${ }^{5}$ sample (Condon et al. 1998) which has:

$$
\left(b(z) \frac{\mathrm{d} N}{\mathrm{~d} z}\right)_{\mathrm{NVSS}}=b_{\mathrm{eff}} \frac{\alpha^{\alpha+1}}{z_{*}^{\alpha+1} \Gamma(\alpha)} z^{\alpha} \mathrm{e}^{-\alpha z / \mathrm{z}_{*}}
$$

where $b_{\text {eff }}=1.98, z_{*}=0.79$ and $\alpha=1.18$ (Ho et al. 2008). The NVSS catalog includes the North sky of -40 deg declination in $1.4 \mathrm{GHz}$ continuum band (Condon et al. 1998). For other observed samples released by different surveys, we should use proper redshift distribution functions as reported e.g. in (McDonald et al. 2005; Giannantonio et al. 2008; Douspis et al. 2008; Ho et al. 2008; Xia et al. 2009; Wang et al. 2010a; Ferraro et al. 2015; Ade et al. 2016c).

The ISW cross-power spectrum is show in Fig. 8. From observational point of view, the value of $\mathcal{C}_{T g, \ell}^{\mathrm{ISW}}$ shows a considerable magnification compared to $\mathcal{C}_{T T, \ell}^{\mathrm{ISW}}$. In the upper panel of Fig. 8, we show the effect of bulk viscosity on the ISW-cross power spectrum. By increasing $\gamma$, the value of the ISW-cross power spectrum goes down and we have a turning point in which the behavior of the ISW-cross power spectrum changes in opposite way but not for all multipoles as revealed by the mentioned figure. For $\gamma \sim \gamma_{\times}$, at the late time, we have a deficiency in $\mathrm{d} Q(a) / \mathrm{d} a$ (Fig. 5), while for almost long interval of scale factor, it gets higher value compared to other cases yielding non-monotonic behavior for ISW cross-power spectrum with galaxies. This behavior provides an opportunity to get more consistent theoretical predictions with relevant observations. The lower panel of Fig. 8 illustrates the $\mathcal{C}_{T g, \ell}^{\mathrm{ISW}}$ in $\mu \mathrm{K}$ scale compared to the NVSS (Ho et al. 2008).

In the next section, we will examine the observational consistency of BVDE model based on observational quantities derived in the perturbations approached.

\section{OBSERVATIONAL CONSISTENCY}

In our previous paper, we mainly concentrated on the background evolution and derived the best fit values for model free parameters. To make more complete present discussion on the contribution of bulk viscous dark energy model in the dark matter perturbations evolution, we focus on an observable quantity coming from perturbation formalism, namely $f \sigma_{8}(z)$. Table 1 contains the observed value of $f \sigma_{8}(z)$ with associated $1 \sigma$ uncertainty according to the "Gold-2017" catalogue (Nesseris et al. 2017, see references therein). Our relevant model free parameters are $\gamma$ and $\Omega_{\mathrm{m}}^{0}$ with constant priors. The Hubble parameter at present time is mainly constrained by $\mathrm{SNIa}+\mathrm{BAO}+$ Planck reported by Mostaghel et al. (2017).

In order to compare the observational data set with that of predicted by our model, we utilize likelihood function with the following $\chi^{2}$ :

$\chi_{\mathrm{RSD}}^{2} \equiv \Delta f \sigma_{8}^{\mathrm{t}} \cdot C^{-1} \cdot \Delta f \sigma_{8}$

5 http://www.cv.nrao.edu/nvss/

\begin{tabular}{ccc}
\hline Index & Redshift & $f \sigma_{8, \mathrm{obs}}$ \\
\hline \hline 1 & 0.02 & $0.428 \pm 0.0465$ \\
2 & 0.02 & $0.398 \pm 0.065$ \\
3 & 0.02 & $0.314 \pm 0.048$ \\
4 & 0.10 & $0.370 \pm 0.130$ \\
5 & 0.15 & $0.490 \pm 0.145$ \\
6 & 0.17 & $0.510 \pm 0.060$ \\
7 & 0.18 & $0.360 \pm 0.090$ \\
8 & 0.25 & $0.3512 \pm 0.0583$ \\
9 & 0.32 & $0.384 \pm 0.095$ \\
10 & 0.37 & $0.4602 \pm 0.0378$ \\
11 & 0.38 & $0.440 \pm 0.060$ \\
12 & 0.44 & $0.413 \pm 0.080$ \\
13 & 0.59 & $0.488 \pm 0.060$ \\
14 & 0.60 & $0.550 \pm 0.120$ \\
15 & 0.60 & $0.390 \pm 0.063$ \\
16 & 0.73 & $0.437 \pm 0.072$ \\
17 & 0.86 & $0.400 \pm 0.110$ \\
18 & 1.40 & $0.482 \pm 0.116$ \\
\hline
\end{tabular}

Table 1. The current observational value of the $f \sigma_{8}(z)$ according to the "Gold-2017" catalogue (Nesseris et al. 2017).

\begin{tabular}{cc}
\hline \hline Parameter & RSD \\
\hline$\Omega_{\mathrm{m}}^{0}$ & $0.303_{-0.038-0.070}^{+0.044+0.093}$ \\
\hline$\gamma$ & $0.033_{-0.033-0.033}^{+0.098+0.182}$ \\
\hline$\sigma_{8}$ & $0.769_{-0.089-0.154}^{+0.080+0.181}$ \\
\hline
\end{tabular}

Table 2. Best fit values for BVDE model using RSD data at $68 \%$ and $95 \%$ confidence intervals.

where $\Delta f \sigma_{8} \equiv f \sigma_{8}^{\text {obs }}(z)-f \sigma_{8}^{\text {the }}\left(z ; \Omega_{\mathrm{m}}^{0}, \gamma\right)$ and $C$ is the covariance matrix of RSD data set. The best fit values for BVDE free parameters based on RSD observation are reported in Table 2. Fig. 9 indicates the behavior of $f \sigma_{8}$ as a function of redshift for various values of model free parameters. The symbols correspond to the most new catalog including observational values. The marginalized likelihood function for $\sigma_{8}, \Omega_{\mathrm{m}}^{0}$ and $\gamma$ determined by RSD observations are depicted in the upper panel of Fig. 10. The lower panel of Fig. 10 indicates the contour plots illustrating the marginalized confidence regions at $68 \%$ and $95 \%$ levels. Taking into account the bulk viscosity for dark energy component, manipulates the growing of the dark matter in the Universe decreasing tension in the present fluctuation spectrum, $\sigma_{8}$, which has been mentioned in (Ade et al. 2016a; Heymans et al. 2012; Erben et al. 2013).

\section{SUMMARY AND CONCLUSION}

In this paper, following our previous paper on proposing a new dynamical dark energy model inspired by thermodynamical dissipative phenomena, we examined the linear perturbation theory of the dark matter in the presence of viscous dark energy model. In order to probe the dark energy properties, the clustering of large scale structure and ISW have been elucidated.

Taking into account viscosity coefficient for our BVDE model, suppresses the $\delta_{\mathrm{m}}^{+}$comparing to the same quantity 


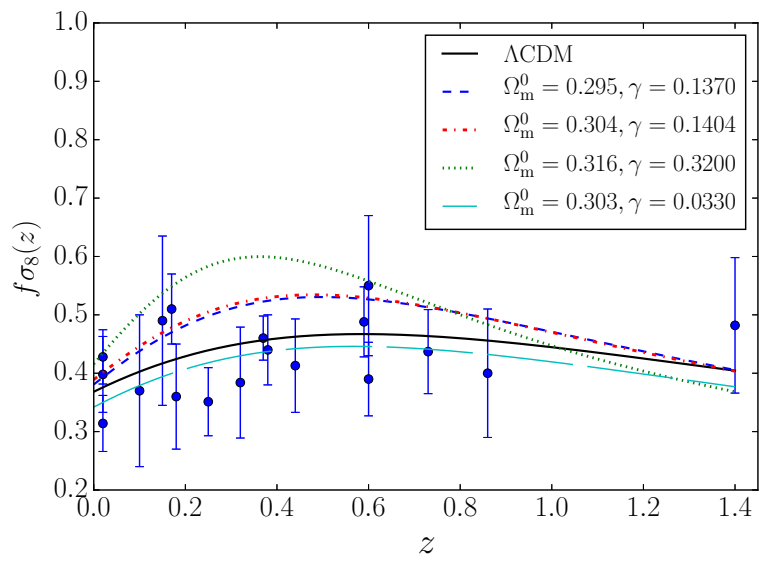

Figure 9. The $f \sigma_{8}$ as a function of redshift for various values of BVDE model free parameters. The solid line corresponds to the best fit parameters based on $\Lambda \mathrm{CDM}$. Increasing the viscous coefficient $(\gamma)$ shows ups and downs in the behavior of $f \sigma_{8}$ leading to have more consistency with observational data points.

computed for $\Lambda$ CDMat early epoch. While dark matter growing mode is boosted at the late time. For higher value of the viscous coefficient, we obtained higher value of scale factor for which the $\delta_{\mathrm{m}}^{+}$becomes higher than $\Lambda$ CDM (see Fig. 2). As illustrated in Fig. 2, the dark matter growing mode is not monotonic function versus scale factor and structure formation experiences a delay in the presence of viscous dark energy.

Fig. 3 indicated the value of growth function $\left(D_{\mathrm{m}}^{+}\right)$. By increasing the bulk viscosity coefficient in the BVDE model, we found a deviation from the standard $\Lambda$ CDM model. Therefore we expect to have a manipulation in the $\sigma_{8}$ introduced by Eq. (14). The linear growth rate in Fig. 4 indicated that for almost $\gamma \lesssim \gamma_{\times}$(Mostaghel et al. 2017), the early dark energy contribution can not compensate the higher role of cold dark matter at the late time. Consequently, the growth rate is higher than $\Lambda$ CDM. Such behavior is no longer valid for almost $\gamma \gtrsim \gamma_{\times}$. We found a bump in $f(z)$ around $z \sim 0.8$ for higher $\gamma$.

ISW power spectrum for our BVDE model has been illustrated in Fig. 7 confirming non-monotonic contribution of viscosity effect in the BVDE model. To give a physical interpretation for this behavior, we should look at the contribution of $\Omega_{\mathrm{DE}}, H, D_{\mathrm{m}}^{+}(a), Q(a)$ and $\mathrm{d} Q(a) / \mathrm{d} a$. The bulk viscosity parameter can extensively modify the rate of Hubble expansion and consequently, it mainly affects the evolution of the dark matter density parameter and $D_{\mathrm{m}}^{+}(a)$. In another word, incorporating bulk viscosity for our dark energy fluid, reduces $\Omega_{\mathrm{DE}} / \Omega_{\mathrm{m}}$ for long period of cosmic evolution. According to Fig. 2, the growing mode of matter is higher than $\Lambda$ CDM. The source term in ISW is diminished considerably leading to have more static gravitational potential and therefore less ISW. This behavior is not monotonic with respect to $\gamma$. For almost $\gamma \gtrsim \gamma_{\times}$, at intermediate range of scale factor, the BVDE model effectively reduces the structure formation giving higher ISW term. It is worth mentioning that, such magnification can never compensate the sharp reduction in $W_{T}^{2}$ and finally we get the lower ISW value comparing to the $\Lambda$ CDM model (Figs. 5, 6 and 7). Indeed, incorporating viscosity for $\gamma \lesssim \gamma_{\times}$damps the growing mode of the newtonian potential slower than $\Lambda \mathrm{CDM}$ model.

To magnify the amount of ISW signal in one hand and on the other hand to reduce the degeneracies in determining the source of fluctuations in the power spectrum, we computed the ISW cross-correlation with visible galaxy density contrast. Such cross-correlation can magnify the value of $\mathcal{C}_{T g, \ell}^{\text {ISW }}$ compared to $\mathcal{C}_{T T, \ell}^{\text {ISW }}$. In BVDE model, the value of the ISW-cross power spectrum decreases by increasing $\gamma$ and we found a turning point confirming non-monotonic behavior of ISW-cross power spectrum. Fig. 8 illustrates the $\mathcal{C}_{T g, \ell}^{\mathrm{ISW}}$ in $\mu \mathrm{K}$ scale compared to the NVSS.

To examine the observational consistency, we have utilized "Gold-2017" RSD observations. The value of $f \sigma_{8}$ as a function of redshift has been indicated in Fig. 9. Our posterior analysis indicated that $\Omega_{\mathrm{m}}^{0}=0.303_{-0.038-0.070}^{+0.044+0.093}$, $\gamma=0.033_{-0.033-0.033}^{+0.098+0.182}$ and $\sigma_{8}=0.769_{-0.089-0.154}^{+0.080+0.181}$ at $1 \sigma$ and $2 \sigma$ level of confidences, respectively. It seems that our model could reduce the tension in $\sigma_{8}$ (see Fig. 10).

Finally, considering the contribution of coupling between dark sectors of the Universe incorporating viscosity for the dark energy could be interesting and utilizing structure formation with background observations would be able to distinguish between such families of models (Schäfer 2008b). Taking into account such interaction may provide an opportunity to examine the stability of underlying viscous dark energy model. To resolve acausal problem for the bulk viscous dark energy fluid, one can keep the collision time scale in the transport equation. We will address them in the future work.

\section{ACKNOWLEDGEMENTS}

We are really grateful to Björn Malte Schäfer for his very constructive comments. We also thank to Nima Khosravi and Marzieh Farhang for their suggestions on the framework of this paper. SMSM is grateful to school of Physics, Institute for research in fundamental sciences (IPM), where some parts of this paper have been finalized.

\section{REFERENCES}

Acquaviva V., Baccigalupi C., 2006, Physical Review D, 74

Ade P. A. R., et al., 2016a, Astron. Astrophys., 594, A13

Ade P. A. R., et al., 2016b, Astron. Astrophys., 594, A14

Ade P. A. R., et al., 2016c, Astron. Astrophys., 594, A21

Afshordi N., 2004, Physical Review D, 70, 083536

Afshordi N., Loh Y.-S., Strauss M. A., 2004, Physical Review D, 69,083524

Amendola L., 1993, Phys. Lett., B301, 175

Amendola L., et al., 2013, Living Rev. Rel., 16, 6

Amendola L., Ballesteros G., Pettorino V., 2014 , Physical Review D, 90

Antolini C., Martinelli M., Fantaye Y., Baccigalupi C., 2013, Journal of Cosmology and Astroparticle Physics, 2013, 024

Baldi M., Pettorino 2011, Monthly Notices of the Royal Astronomical Society: Letters, 412, L1

Barbosa C., Velten H., Fabris J., Ramos R. O., 2017, Physical Review D, 96, 023527 

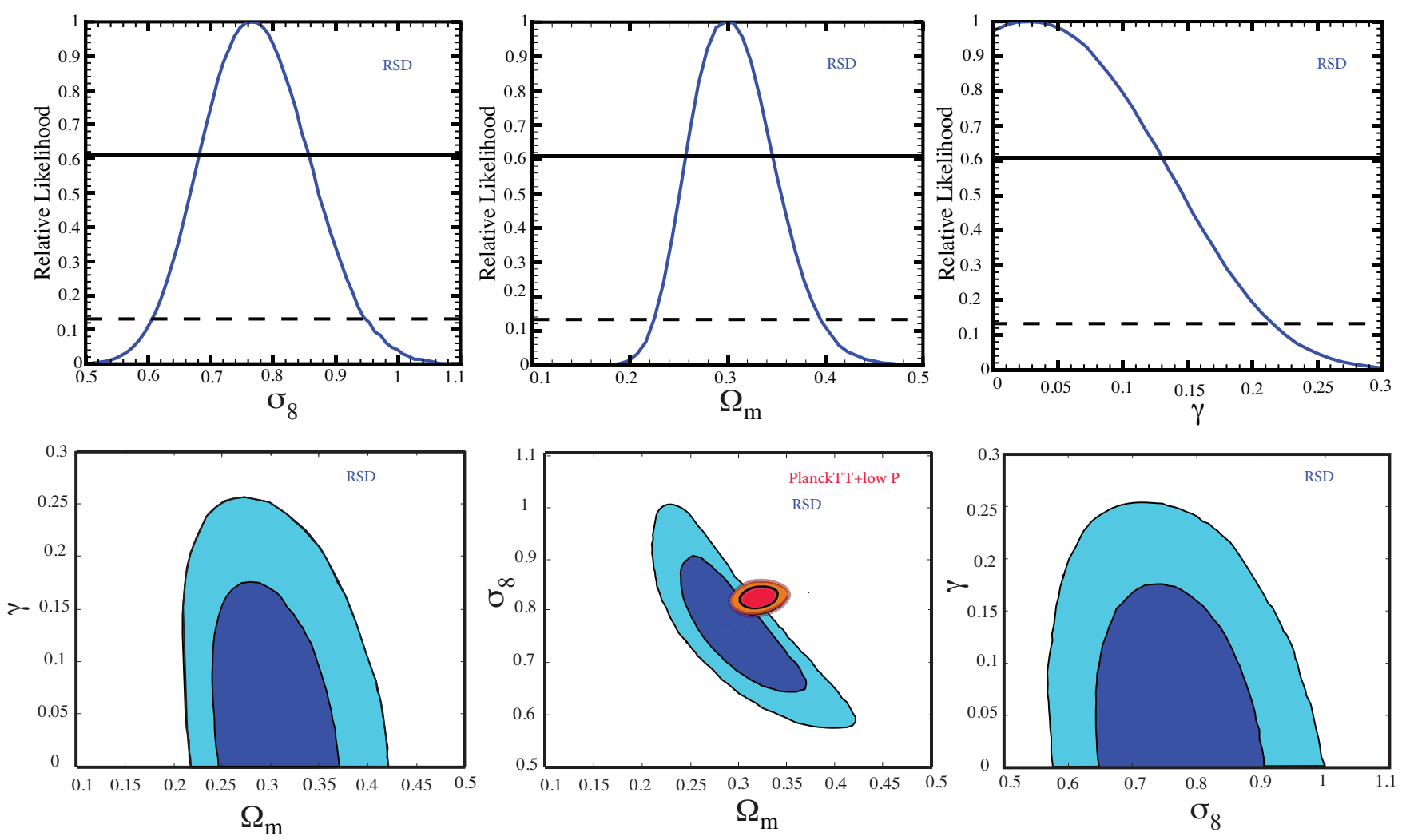

Figure 10. , Upper panels corresponds to marginalized posterior function for BVDE model free parameters. Marginalized confidence regions at $68 \%$ and $95 \%$ confidence levels. The small contours is devoted to $\Lambda$ CDM model.

Bardeen J. M., 1980, Physical Review D, 22, 1882

Bardeen J. M., Bond J. R., Kaiser N., Szalay A. S., 1986, The Astrophysical Journal, 304, 15

Barrow J. D., Saich P., 1993, Monthly Notices of the Royal Astronomical Society, 262, 717

Bento M. C., Bertolami O., Sen A. A., 2002, Phys. Rev., D66, 043507

Bernal J. L., Verde L., Riess A. G., 2016, Journal of Cosmology and Astroparticle Physics, 2016, 019

Boughn S. P., Crittenden R., 2004, Nature, 427, 45

Boughn S. P., Crittenden R., 2005a, New Astronomy Reviews, 49,75

Boughn S. P., Crittenden R., 2005b, Monthly Notices of the Royal Astronomical Society, 360, 1013

Caldwell R. R., 2002, Phys. Lett., B545, 23

Carbone C., Baldi M., Pettorino V., Baccigalupi C., 2013, Journal of Cosmology and Astroparticle Physics, 2013, 004

Condon J. J., Cotton W. D., Greisen E. W., Yin Q. F., Perley R. A., Taylor G. B., Broderick J. J., 1998, The Astronomical Journal, 115, 1693

Contaldi C. R., Hoekstra H., Lewis A., 2003, Physical review letters, 90, 221303

Cooray A., 2002, Phys. Rev., D65, 083518

Copeland E. J., Sami M., Tsujikawa S., 2006, Int. J. Mod. Phys., D15, 1753

Crittenden R. G., Turok N., 1996, Physical Review Letters, 76, 575

Dent J. B., Dutta S., Weiler T. J., 2009, Physical Review D, 79, 023502

Disconzi M. M., 2014, Nonlinearity, 27, 1915

Douspis M., Castro P. G., Caprini C., Aghanim N., 2008, Astron- omy \& Astrophysics, 485, 395

Durrer R., Kunz M., Melchiorri A., 1999, Physical Review D, 59

Eckart C., 1940, Phys. Rev., 58, 919

Erben T., et al., 2013, Monthly Notices of the Royal Astronomical Society, 433, 2545

Fan Y., Wu P., Yu H., 2016, Classical and Quantum Gravity, 33, 085006

Fang W., Li B., Zhao G.-B., 2017, Phys. Rev. Lett., 118, 181301

Ferraro S., Sherwin B. D., Spergel D. N., 2015, Physical Review D, 91, 083533

Flender S., Hotchkiss S., Nadathur S., 2013, Journal of Cosmology and Astroparticle Physics, 2013, 013

Fosalba P., Gaztanaga E., Castander F. J., 2003, The Astrophysical Journal Letters, 597, L89

Germani C., Kehagias A., 2010, Phys. Rev. Lett., 105, 011302

Giannantonio T., Scranton R., Crittenden R. G., Nichol R. C., Boughn S. P., Myers A. D., Richards G. T., 2008, Phys. Rev., D77, 123520

Gordon C., Hu W., 2004, Physical Review D, 70, 083003

Granett B. R., Neyrinck M. C., Szapudi I., 2008, The Astrophysical Journal Letters, 683, L99

Heymans C., et al., 2012, Mon. Not. Roy. Astron. Soc., 427, 146

Heymans C., et al., 2013, Monthly Notices of the Royal Astronomical Society, 432, 2433

Hiscock W. A., Lindblom L., 1983, Annals of Physics, 151, 466

Hiscock W. A., Lindblom L., 1985, Phys. Rev., D31, 725

Hiscock W. A., Lindblom L., 1988, Physics Letters A, 131, 509

Hiscock W. A., Salmonson J., 1991, Phys. Rev., D43, 3249

Ho S., Hirata C., Padmanabhan N., Seljak U., Bahcall N., 2008, Physical Review D, 78, 043519

Horndeski G. W., 1974, Int. J. Theor. Phys., 10, 363

Hu W., Sugiyama N., 1994, Phys. Rev., D50, 627

Hu W., White M., 1996, The Astrophysical Journal, 471, 30 
Huterer D., Shafer D. L., 2018, Rept. Prog. Phys., 81, 016901 Israel W., Stewart J., 1979, Annals of Physics, 118, 341 Jou D., Casas-Vázquez J., Lebon G., 2009, Extended Irreversible Thermodynamics. Springer-Verlag $\mathrm{GmbH}$

Kaiser N., 1987, Mon. Not. Roy. Astron. Soc., 227, 1

Kaiser N., 1992, The Astrophysical Journal, 388, 272

Khosravi N., 2016, Phys. Rev. D, 94, 124035

Khosravi N., 2018, Phys. Dark Univ., 21, 21

Khosravi S., Mollazadeh A., Baghram S., 2016, JCAP, 1609, 003

Khosravi N., Baghram S., Afshordi N., Altamirano N., 2017, arXiv preprint arXiv:1710.09366

$\begin{array}{llll}\text { Kodama H., } & \text { Sasaki } & \text { M., }\end{array}$ Progress of Theoretical Physics Supplement, 78, 1

Kofman L., Starobinskij A. A., 1985, Pisma v Astronomicheskii Zhurnal, 11, 643

Könnig F., Nersisyan H., Akrami Y., Amendola L., Zumalacárregui M., 2016, JHEP, 11, 118

Kovács A., Szapudi I., Granett B. R., Frei Z., 2013, Monthly Notices of the Royal Astronomical Society: Letters, 431, L28

Kunz M., Nesseris S., Sawicki I., 2015, Physical Review D, 92

Lahav O., Lilje P. B., Primack J. R., Rees M. J., 1991, Monthly Notices of the Royal Astronomical Society, 251, 128

Landau L. D., Lifshitz E. M., 1987, Course of theoretical physics/by LD Landau and EM Lifshitz, 6

Lesgourges J., 2013, in, Searching for New Physics at Small and Large Scales: TASI 2012. World Scientific, pp 29-97

Lewis A., Challinor A., 2006, Physics Reports, 429, 1

Li B., Barrow J. D., 2009, Phys. Rev., D79, 103521

Limber D. N., 1954, The Astrophysical Journal, 119, 655

Ling C., Wang Q., Li R., Li B., Wang J., Gao L., 2015, Phys. Rev., D92, 064024

Maartens R., 1996, arXiv preprint astro-ph/9609119

Mainini R., Mota D. F., 2011, The Astrophysical Journal, 744, 3

Majerotto E., Sapone D., Schäfer B. M., 2015, Monthly Notices of the Royal Astronomical Society, 456, 109

McDonald P., et al., 2005, The Astrophysical Journal, 635, 761

McDonald P., Trac H., Contaldi C., 2006, Monthly Notices of the Royal Astronomical Society, 366, 547

Mostaghel B., Moshafi H., Movahed S. M. S., 2017, The European Physical Journal C, 77, 541

Mukhanov V., 1992, Physics Reports, 215, 203

Nadathur S., Hotchkiss S., Sarkar S., 2012, Journal of Cosmology and Astroparticle Physics, 2012, 042

Nesseris S., Pantazis G., Perivolaropoulos L., 2017, Phys. Rev. D, 96, 023542

Olivares G., Atrio-Barandela F., Pavón D., 2008, Physical Review D, 77,103520

Padmanabhan N., Hirata C. M., Seljak U., Schlegel D. J., Brinkmann J., Schneider D. P., 2005, Physical Review D, 72,
043525

Peebles P. J. E., 1984, The Astrophysical Journal, 284, 439

Peebles P. J. E., 1993, Principles of Physical Cosmology. Princeton University Press

Peel A., Lin C.-A., Lanusse F., Leonard A., Starck J.-L., Kilbinger M., 2017, Astron. Astrophys., 599, A79

Perlmutter S., et al., 1999, Astrophys. J., 517, 565

Raveri M., Baccigalupi C., Silvestri A., Zhou S.-Y., 2015, Physical Review D, 91

Rees M. J., Sciama D. W., 1968, Nature, 217, 511

Rezzolla L., Zanotti O., 2013, Relativistic hydrodynamics. Oxford University Press

Riess A. G., et al., 1998, Astron. J., 116, 1009

Riess A. G., et al., 2016, The Astrophysical Journal, 826, 56

Sachs R. K., Wolfe A. M., 1967, The Astrophysical Journal, 147, 73

Sapone D., Kunz M., 2009, Physical Review D, 80, 083519

Schäfer B. M., 2008a, Monthly Notices of the Royal Astronomical Society, 388, 1394

Schäfer B. M., 2008b, Monthly Notices of the Royal Astronomical Society, 388,1403

Schäfer B. M., Bartelmann M., 2006, Mon. Not. Roy. Astron. Soc., 369, 425

Schäfer B. M., Douspis M., Aghanim N., 2009, Monthly Notices of the Royal Astronomical Society, 397, 925

Scranton R., et al., 2003, arXiv preprint astro-ph/0307335

Seljak U., Zaldarriaga M., 1996, Astrophys. J., 469, 437

Smith R. E., et al., 2003, Monthly Notices of the Royal Astronomical Society, 341, 1311

Song Y.-S., Percival W. J., 2009, Journal of Cosmology and Astroparticle Physics, 2009, 004

Song Y.-S., Sawicki I., Hu W., 2007, Phys. Rev., D75, 064003

Väliviita J., Majerotto E., Maartens R., 2008, Journal of Cosmology and Astroparticle Physics, 2008, 020

Velten H., Borges H. A., Carneiro S., Fazolo R., Gomes S., 2015, Monthly Notices of the Royal Astronomical Society, 452, 2220

Wang Y. T., Gui Y. X., Xu L. X., Lu J. B., 2010a, Phys. Rev. D, 81,083514

Wang Y., Xu L., Gui Y., 2010b, Physical Review D, 82, 083522

Xia J.-Q., Viel M., Baccigalupi C., Matarrese S., 2009, Journal of Cosmology and Astroparticle Physics, 2009, 003

Zlatev I., Wang L., Steinhardt P. J., 1999, Physical Review Letters, 82, 896

This paper has been typeset from a $\mathrm{T}_{\mathrm{E}} \mathrm{X} / \mathrm{LAT} \mathrm{T}$ file prepared by the author. 